\title{
A Review Literature on UPA for Image and Video Transmission
}

\author{
Monika Vyas ${ }^{1}$, Sumeet Dhillon ${ }^{2}$
}

\begin{abstract}
Unpredictable picture codes can have limit moving toward execution with an iterative BP decoder under AWGN channel with BPSK tweak. Ordinarily in BPSK adjustment, each piece inside the square has a similar power. In this paper, we will attempt to concentrate unequal power portion (UPA) by utilizing BPSK tweak for unpredictable parallel codes of picture. We demonstrate that consistent power adjustment require not prompt the best execution. With UPA we see pick up, with bigger increases conceivable if the recipient knows the points of interest of the UPA strategy. Ideal power portion is depend firmly on the codes. Our work delineates the motivation behind the UPA plot for transmission of picture codes. Comes about present that unequal power assignment system gives critical picture quality change when contrasted with various equivalent power distributions plans. What's more, this system averts mutilation of pictures and recordings.
\end{abstract}

Keywords: JSCC, OFDM, Unequal power allocation (UPA), BER (Bit error rate), Power Optimization

\section{Introduction}

Conventionally, source and channel codes are designed separately and then cascaded together. Provided with an infinite degree of complexity, source and channel coding can be separated without sacrificing fidelity.

However, when many source coding techniques are applied, some coded bits (e.g., the most significant bit of a scalarquantized signal) are far more important than others. In practice, unequal error protection by some means, for example, those in cellular systems, is provided to improve the system performance. Power allocation, especially suitable for a multicarrier modulation system distributes the available quota of transmission power to different bits according to their importance in order to provide the best output quality at the receiver output. Power allocation was described for pulse-coded modulation, for vector quantizes, for image transmission. It was shown that power allocation can be applied for multicarrier modulation or its generalized version.

Orthogonal Frequency Division Multiplexing (OFDM), a type of multicarrier modulation, is the standard modulation scheme for digital audio broadcasting (DAB) and is also proposed for digital television. In this paper, we demonstrate by simulation that power allocation in OFDM can be used to provide unequal error protection for gradual quality degradation as the channel becoming noisier. While the design of the whole working system is not provided, some numerical results show significant improvement of OFDM over single-carrier systems. In the remaining part of this paper, we will first review method and algorithm on power allocation in OFDM system for unequal error protection provisioning. We will also review digital audio compression scheme.

Future radio mobile communication systems that can provide diverse transmission services, such as video, image and data, with high transmission rates and low transmitted power are of much interest. The problem of transmitting high data rates over frequency selective fading channel is inter symbol interference, ISI, which merely degrades the presentation of the system. Multicarrier transmission in the way of orthogonal frequency division multiplexing (OFDM) is a possible solution that can combat ISI effectively. The OFDM technique has many advantages in wireless communications and is used in many practical systems. OFDM allows digital data to be efficiently and reliably transmitted in multipath environments by lowering of the symbol rate resulting in lowering of the Inter symbol Interference (ISI). In OFDM scheme; a single high-rate

Bit stream is converted to low-rate parallel bit streams. Parallel streams are modulated onto orthogonal sub- carriers. Spectrum of these sub-carriers are closely spaced and overlapped to achieve high bandwidth efficiency. The bandwidth of these sub-carriers becomes small compared with the coherence bandwidth of the channel; i.e. the individual sub-carriers experience only flat fading. So, OFDM transforms the frequency selective fading channel into multiple independent flat fading sub-channels. Therefore, an OFDM system can achieve a high data rate and a reliable transmission in a fading channel. OFDM also uses a cyclic guard time at the start of each symbol to remove any ISI shorter than its length.

\section{Unequal Power Allocation Techniques for Image Transmission}

Transmission of progressive images, such as coded through the set partition in hierarchical tree, is often desired, because the restored image quality can be incrementally improved and is always the optimal for a given number of sequentially decoded error free bits. However, progressive data are sensitive to the channel noise. A single bit error may cause the loss of synchronization between the encoder and the decoder and, hence, makes the data completely useless. JSCC ( joint source channel coding) is the most commonly studied joint design problem for image and video communication in the literature. Another important joint design problem is that of transmission power allocation and optimization for image and video communication. The main goal for such problems is either to minimize the total distortion with a constraint on available transmission power, or to minimize the power usage with a constraint on maximum "tolerable" distortion. In Section I-A, we discuss

\section{Volume 6 Issue 12, December 2017}




\section{International Journal of Science and Research (IJSR) \\ ISSN (Online): 2319-7064}

Index Copernicus Value (2016): 79.57 | Impact Factor (2015): 6.391

various existing joint design methods for efficient image and video communication.

The main goal of all the methods discussed above was either the minimization of energy/power with a constraint on total allowable distortion, or the minimization of distortion with a constraint on total energy/power. These methods showed large amounts of energy/power savings or quality gains as compared to methods that transmitted the images and videos with equal power.

In [1], Modestino et al. proposed JSCC methods for digital images. In these methods, distortion in the form of meansquared-error (MSE) was computed using the probability density functions of the coded source, the quantizer step size and the channel probability of error. Most important bits were protected using selective error protection. These methods demonstrated that significant increase in image quality could be achieved using efficient channel coding without imposing any penalty on the transmission bandwidth.

In [2], Chande and Farvardin proposed a JSCC scheme for progressive image transmission over noisy channels. They developed algorithms for optimal allocation of source and channel coding bits using average distortion (MSE), average peak-signal-to-noise ratio (PSNR) and average useful source coding rate as the cost functions.

In [3], Sherwood and Zeger proposed an efficient method for progressively coded image transmission using concatenated channel codes.

In [4], Eisenberg et al. presented a transmit power management scheme for transmission of compressed video sequences over a wireless channel. The energy needed to transmit the video was minimized under a delay and distortion constraint. To achieve this, the source coding and physical layer parameters were adjusted simultaneously. Their results showed that it is more energy efficient to jointly optimize the source coding parameters and transmission power than adjusting them independently.

In [5], Atzori presented a method for unequal power distribution among different JPEG2000 coding units based on their contribution to total image quality. In this scheme, the JPEG2000 stream was divided into different coding packet groups. These different groups were transmitted through separate sub channels and different rate and power. This scheme showed a PSNR gain of around $4 \mathrm{~dB}$ at low SNRs for additive white Gaussian noise (AWGN) and Rayleigh fading channels as compared to equal power allocation.

In [6], Kozintsev and Ramchandran presented a multi resolution framework for optimally matching the source resolution and signal constellation resolution trees for a wavelet image decomposition based source coding model. The multiple resolutions resulting from sub band decomposition of the image were mapped to the multi resolution channel codes based on instantaneous channel state information (CSI). This was achieved using a Lagrangian-based optimization formulation while keeping the transmitted modulation energy and bandwidth fixed. It was shown that using the multiresolution based approach, 2$3 \mathrm{~dB}$ of gain in signal-to-noise ratio (SNR) is typically achieved over source-channel optimized single resolution based approaches.

In [7] Zhang et al. presented a power minimized bit allocation scheme for wireless video communication. In this paper, the authors allocated the total available bits between the source and the channel coders based on wireless channel condition and video quality requirements such that the total power consumption was minimized.

In [13], Yousefi'zadeh et al. presented a power optimization problem for wireless multimedia transmission with space time block codes. A set of optimization problems aimed at minimizing the total power consumption with a given level of quality of service and bit budget were formulated. They used Gauss-Markov and video source models as their source coding model, Rayleigh fading channel with Bernoulli/Gilbert-Elliott loss models, and space-time codes for transmission. Their results showed that lowest optimal power values were obtained when multiple transmit and receive antennas were used.

In [14], Lu et al. developed a power minimization method subject to a given level of quality of service for $\mathrm{H} .263$ video encoder employing Reed-Solomon channel codes for transmission. They used empirical models to estimate the distortion due to source coding and transmission errors. They minimized the total power consumption of the system consisting of power consumption by the source and the channel encoder, and the transmission power, with a constraint on total allowable distortion.

Kim and Kim presented another H.263 based power optimization method for code division multiple access (CDMA) systems in [15]. In this method, a distortion model that takes motion compensation into account was developed for H.263 video data employing error concealment. This model was then used to minimize the target bit error rate (BER) of image frames such that the total consumed power is minimized with a constraint on maximum distortion. This scheme showed around 3.5 dB PSNR gain as compared to conventional schemes that use fixed target BER.

\section{Power Optimization for Video Transmission Over OFDM}

In this section we explain our result to practical implementation and assume video signal compressed by H.263 encoder, channel encoded by an RS channel code and transmitted through a wireless channel. We consider a slowly fading channel whose coherence time is large, since we only consider the path loss and additive noise. We consider DPSK is modulation technique. In an H.263 encoder, each macro block in frame can be encoded in either the INTRA mode or the INTER mode. In the INTRA mode, a macro block is directly transferred using (DCT) Discrete Cosine Transform and DCT coefficients are entropy coded and quantized. In an INTER mode, a macro block is protected from previously encoded frame using motioncompensated prediction, and the INTRA mode method is

\section{Volume 6 Issue 12, December 2017}

\section{www.ijsr.net}




\section{International Journal of Science and Research (IJSR) \\ ISSN (Online): 2319-7064}

Index Copernicus Value (2016): 79.57 | Impact Factor (2015): 6.391

used for prediction of error. All macro blocks are coded in the INTRA mode, a macro block in frames is generally coded in INTER mode, if the prediction is undetermined, or if it is forced to be coded in INTRA mode for error-detection purpose. Avery important encoder parameter is the INTRA rate, which manages how often a macro block is forced to be coded in the INTRA mode. The rate control algorithm in the encoder can regulate the bit rate by adjusting the quantization parameter and the coded frame rate according to the status of the encoder buffer. From the user view, a user can specify the INTRA rate and the encoding frame rate fs, and the target bit rate Rs. We can denote the distortion introduced at the source encoder when the bit rate is Rs and the INTRA rate is by Ds.

Note that Ds corresponding to values of gives us operational rate-distortion curves as in, with higher curve corresponding to larger. This is because a larger means more macro blocks are coded using the INTRA mode, which yields higher distortion at the same bit rate than the INTER mode. The total distortion at the decoder Dt is generally higher than Ds due to transmission errors. We define $\mathrm{Dv}=\mathrm{DtDs}$ as the additional distortion incurred by transmission errors. This additional distortion depends on the channel error characteristics, the channel coder, the error resilience, as well as transmission power the of the source coder. We assume that the bits contained in each RS coded block correspond to one packet. We further assume a CRC code is inserted to each and every packet, so that any packet which contains un-correctable channel errors can be found. We can use $\mathrm{pL}$ to present the packet loss rate after decoding of RS channel, which depends on the code rate $r$ and the energy of transmission per bit for channel parameters, for eg noise level and the distance between destination and mobile. The error resilience of the H.263 encoder depends on the INTRA rateand other error- recovery tools conveyed. We will assume that all other error-resilience so that the error resilience of the coded bit stream is controlled solely by with the above assumptions, Dv can be abstracted as a function of and $\mathrm{pL}$ only. To solve the power optimization problem of, we also need to model the power consumptions of the transmitter, channel coder and the video coder. One of the contributions of this paper is the development and validation of power consumption models of H.263 and RS coders. Using power consumption models and the distortion, the total power consumption at the transmitter can be Reduced by adjusting the source and channel coder parameters, and the transmit energy Eb, for a fixed set of channel parameters. Power savings that result from this optimization.

\section{Conclusion}

In this paper, we have given an unequal power allocation technique for the transmission of JPEG compressed images and videos over OFDM (orthogonal frequency division multiplexing). The image is divided into different streams with unequal contribution to total image quality. These different streams were transmitted using different antennas with unequal power which minimizes the distortion in the transmitted image. The total transmit power is kept constant at any given instant. We also presented unequal power allocation problem. Results show that our unequal power allocation scheme provides significant gains in terms of
PSNR over various equal power allocation schemes. To the best of our knowledge no unequal power allocation technique exists for image transmission over MIMO systems. We can extend this work to different video coding schemes and advanced space-time coding technologies.

\section{References}

[1] Y.Wang, S.Wenger, J.Wen, and A. Katsaggelos, "Error resilient video coding techniques,'IEEE Signal Process, vol. 17, no. 4, pp. 61-82, Apr. 2010.

[2] A. J. Goldsmith and M. Effros, "Joint design of fixedrate source codes and channel codes," IEEE Trans., vol. 46, no. 10, pp. 1301-1312, Oct. 1998.

[3] N. Farvardin and V. Vaishampayan, "Optimal quantizer for noisy channel: An approach to combined sourcechannel coding,'IEEE Trans. Inf. Theory, vol. IT-33, no. 11, pp. 827-838, Nov. 1987.

[4] P. Sherwood and K. Zeger, "Error protection for progressive image transmission over memoryless and fading channels," IEEE Trans.Commun., vol. 46, no. 12, pp. 1555-1559, Dec. 2006.

[5] J. W. Modestino and D. G. Daut, "Combined sourcechannel coding for images," IEEE Trans. Commun., vol. COM-27, no. 11, pp. 1644-1659, Nov. 1979.

[6] Sabir, M.F., Sheikh, H.R., Heath, R.W., Jr., and Bovik, A.C. "A joint source-channel distortion model for JPEG compressed images", IEEE Transactions on Image Processing, Vol.15, No.6, pp.1349-1364, 2006.

[7] Sabir, M.F., Bovik, A.C. "Unequal Power Allocation for JPEG Transmission Over MIMO Systems", IEEE Transactions on Image Processing, Vol. 19, No. 2, pp. 410 - 421, 2010.

[8] Alamouti, S.M. "A simple transmit diversity technique for wireless communications", IEEE Journal, Vol. 16, No. 8, pp. 1451- 1458, 1998.

[9] Paulraj, A., Nabar, R. and Gore, D. "Introduction to Space- Time Wireless Communications", Cambridge University Press, 2009.

[10] Simon, M.K. and Alouini, M.S. "Digital Communication over Fading Channels: A Unified Approach to Performance Analysis", WileyInterscience, 2001.

[11] Jafarkhani, H. "Space-Time Coding: Theory and Practice", Cambridge University Press, 2006.

[12] M. Ghanbari and V. Seferidis, "Cell loss concealment in ATM video codecs,'IEEE Trans. Circuits Syst. Video Technol., vol. 3, pp. 238-247, July 1995.

[13] J.W. Park, J.W. Kim, and S. U. Lee, "DCT coefficients recovery-based error concealment scheme and its application to the MPEC-2 bit stream error,'IEEE Trans. Circuits Syst. Video Technology., vol. 7, pp. 845-854, Dec. 1997.

[14] J. Perez-Romero, L. G. Alonso, “Average block error probability in the reverse link of a packet CDMA system under Rayleigh fading channel condition,'IEEE Commun. Lett., vol. 4, pp. 116-118, Apr. 2000.

[15] Sallent and R. Agusti, "A proposal for an adaptive SALOHA access system for a mobile CDMA environment," IEEE Trans,vol. 47, pp. 977-986, Aug. 2000.

[16] S. Lin and D. J. Costello, "Error Control Coding: Fundamentals and Applications". Englewood Cliffs, NJ:

\section{Volume 6 Issue 12, December 2017}




\section{International Journal of Science and Research (IJSR)}

ISSN (Online): 2319-7064

Index Copernicus Value (2016): 79.57 | Impact Factor (2015): 6.391

Prentice-Hall, 1983.

[17] K. J. Gilhousen, I. M. Jacobs, R. Padovani, A. J. Viterbi, I. A. Weaver Jr, and C. E. Wheatly III, "On the capacity of a cellular CDMA system,'IEEE Trans. Veh. Technol., vol. 40, pp. 303-312, May 1991.

[18] A. J. Viterbi, CDMA, "Principles of Spread Spectrum." Reading, MA: Addison-Wesley, 1995.

[19]S. B. Wicker, "Error Control Systems for Digital Communication Storage", Englewood Cliffs, 2000

Volume 6 Issue 12, December 2017

www.ijsr.net

Licensed Under Creative Commons Attribution CC BY 